\title{
Late presentation of a complete complex thumb metacarpophalangeal joint dislocation: A case report
}

\author{
Arash Izadpanah BSc, Kyle Wanzel MD MEd FRCSC
}

\begin{abstract}
A Izadpanah, K Wanzel. Late presentation of a complete complex thumb metacarpophalangeal joint dislocation: A case report. Can J Plast Surg 2011;19(4):139-142.

Metacarpophalangeal (MP) joint injuries and dislocations of the fingers and thumb are not uncommon. They can be classified directionally as either being volar or dorsal, and are further categorized as incomplete, simple complete or complex complete. Complex dislocations are described as dislocations that are irreducible and often require surgical intervention. This is often because tissue has become entrapped within the MP joint, precluding its anatomical reduction. For the thumb MP joint, anatomical structures that may become trapped include the volar plate, sesamoid bones, bony fracture fragments or the flexor pollicis longus tendon. Both dorsal and volar surgical approaches have been described, and their relative merits will be discussed. The unusual case of a late presentation (two months postinjury) of a complex complete dorsal dislocation of the thumb MP joint approached from a dorsal incision is presented.
\end{abstract}

Key Words: Complex joint dislocation; Irreducible dislocation; Metacarpophalangeal joint; Open reduction; Surgical approach
Metacarpophalangeal (MP) joint injuries and dislocations of the Ifingers and thumb are not uncommon (1-8). They can be classified directionally as either being volar or dorsal, although volar dislocations of the thumb MP joint are exceedingly rare (4). Dorsal dislocations are commonly classified with respect to their ease of reduction into three different categories: incomplete, simple complete and complex $(4,6)$.

Incomplete dislocations are better described as subluxations because a portion of the joint remains congruous. The collateral ligaments remain intact, the joint is easily reduced and it is stable on postreduction testing. Complete dislocations describe complete disassociation of the joint, and indicate a significant injury or rupture to the volar plate, joint capsule and at least part of the collateral ligaments. In simple complete dislocations, a closed reduction is expected to be relatively easy. In comparison, complex complete dislocations are described as dislocations that are not easily reducible and often require surgical intervention. Failure to achieve a closed reduction is usually due to the interposition of the volar plate, sesamoid bones, bony fracture fragments or the flexor pollicis longus tendon within the MP joint space, precluding its reduction.

In a complex complete, irreducible thumb MP joint dislocation, the most common culprit is an entrapped volar plate. It usually ruptures from its weakest proximal attachments to the metacarpal bone, remains attached to the base of the proximal phalanx, and then resides within the joint space trapped between the base of the proximal phalanx and the head of the metacarpal. This hinders the reduction of dislocated joint and necessitates an operative approach (8-19).

We present a case of a complex complete, dorsal, thumb MP joint dislocation in a patient presenting two months postinjury. To our knowledge, this is the only case of chronic complex thumb MP joint dislocation that is described in the literature.

\section{La présentation tardive d'une dislocation métacarpophalangienne complexe complète du pouce : un rapport de cas}

Les lésions des articulations métacarpophalangiennes (MP) et les dislocations des doigts et du pouce ne sont pas rares. On peut les classer selon leur orientation palmaire ou dorsale ainsi que comme incomplètes, simples complètes ou complexes complètes. Les dislocations complexes sont décrites comme des dislocations impossibles à réduire, qui exigent souvent une intervention chirurgicale parce les tissus sont coincés dans l'articulation MP, ce qui en empêche la réduction anatomique. Pour ce qui est de l'articulation MP du pouce, la plaque palmaire, les os sésamoïdes, des fragments osseux de fracture et le tendon long fléchisseur du pouce peuvent se trouver coincés. Il existe des méthodes chirurgicales dorsales ou palmaires, et leurs avantages relatifs sont exposés. Les auteurs présentent le cas inhabituel d'un patient qui a consulté à cause d'une dislocation dorsale complète de l'articulation MP du pouce deux mois après sa survenue, laquelle a été traitée par incision dorsale. 

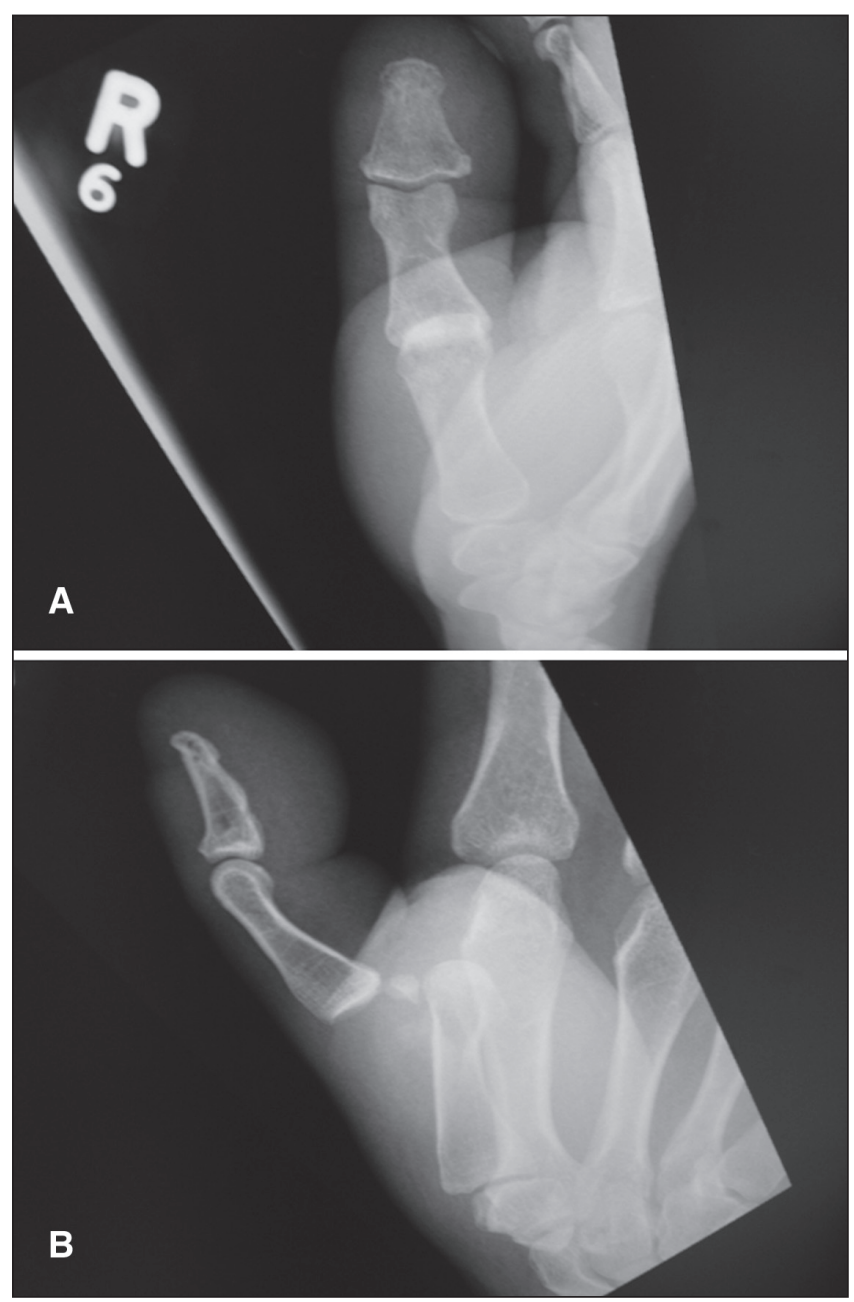

Figure 1) Right thumb anteroposterior (A) and lateral (B) radiographs at presentation demonstrating a complex complete dorsally dislocated metacarpophalangeal joint. Note the excessively widened metacarpophalangeal joint space and possible sesamoid bone within the joint space on the lateral $x$-ray

extensor pollicis longus and brevis tendons. An extensive network of mature scar tissue was encountered, encasing the dorsal joint capsule, base of the proximal phalanx and collateral ligamentous structures. Cautious debridement and dissection was undertaken. The base of the proximal phalanx was clearly identified and delineated, as were portions of the ruptured joint capsule and surrounding structures.

The proximal end of the volar plate was then identified and dissected. It was found to be lying on the dorsal surface of the metacarpal head, trapped between the head of the metacarpal volarly and the base of the proximal phalanx dorsally. A longitudinal incision in the volar plate, from proximal end to distal, was then performed (Figure 2). The leaflets of the volar plate were then allowed to sublux radially and ulnarly around the metacarpal head and slip back to their anatomical position, volar to the metacarpal head. On the radial aspect of the MP joint, the radial sesamoid bone was found within the joint. This was freed and directed radially out of the joint space. A Freer elevator was then passed over the metacarpal head to distract open the MP joint space and gentle, distally directed pressure was applied to the base of the proximal phalanx to reduce the MP joint. After clearing both the sesamoid and the volar plate from the joint space, and given that the dislocation was two months old, it was surprisingly easy to mobilize and reduce the joint. After obtaining and maintaining the desired reduction, stability of the joint was tested and found to be quite lax. Two crossed K-wires were then applied for stability, with the MP joint

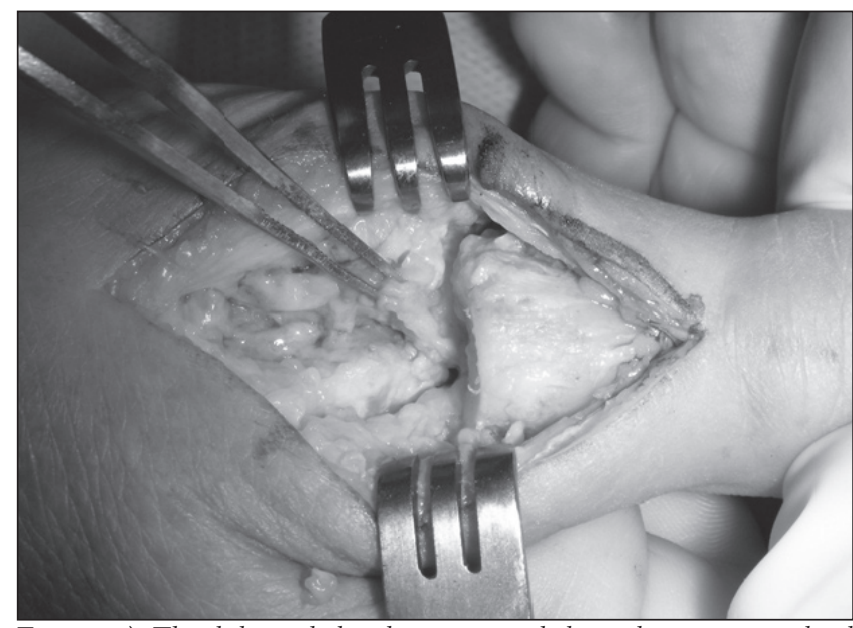

Figure 2) The dislocated thumb metacarpophalangeal joint is visualized through a dorsal approach. Note the base of the proximal phalanx lying completely dorsal to the metacarpal head. The entrapped volar plate is seen between the two bones and has been split longitudinally, with the radial half being grasped with forceps

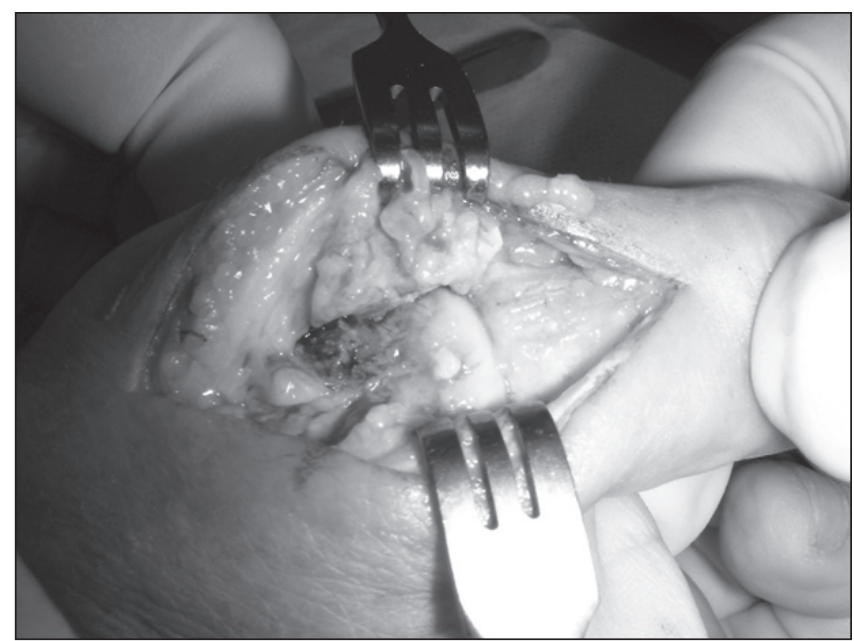

Figure 3) The thumb metacarpophalangeal joint was reduced after releasing the entrapped volar plate, splitting it longitudinally and allowing it to slip back to its anatomical position volar to the metacarpal head

held in slight flexion (Figure 3). Intraoperative fluoroscopy confirmed an anatomic reduction (Figure 4).

The joint capsule was closed using 4-0 vicryl sutures. The extensor apparatus was similarly reapproximated with 4-0 vicryl and the skin closed with 4-0 nylon. The patient was then placed into a temporary plaster splint and the tourniquet deflated.

\section{DISCUSSION}

Complex MP joint dislocations are classically described as complete. irreducible dislocations, and require a surgical approach for reduction and proper alignment. They occur most commonly in the index and little fingers. They are relatively rare in the thumb, and exceedingly uncommon in the long or ring fingers $(4,7)$. The most common structure that inhibits a closed reduction of a complex MP joint dislocation is the volar plate (20). It usually ruptures from its weakest proximal attachments to metacarpal bone, remains attached to the base of the proximal phalanx, and flips over the metacarpal head, becoming trapped between the base of the proximal phalanx dorsally and the head of the metacarpal volarly. Any attempts at reducing the proximal phalanx over the metacarpal head are then impossible because the volar plate remains wedged within the joint space. Other culprits reported to lodge within the joint and preclude reduction include the 

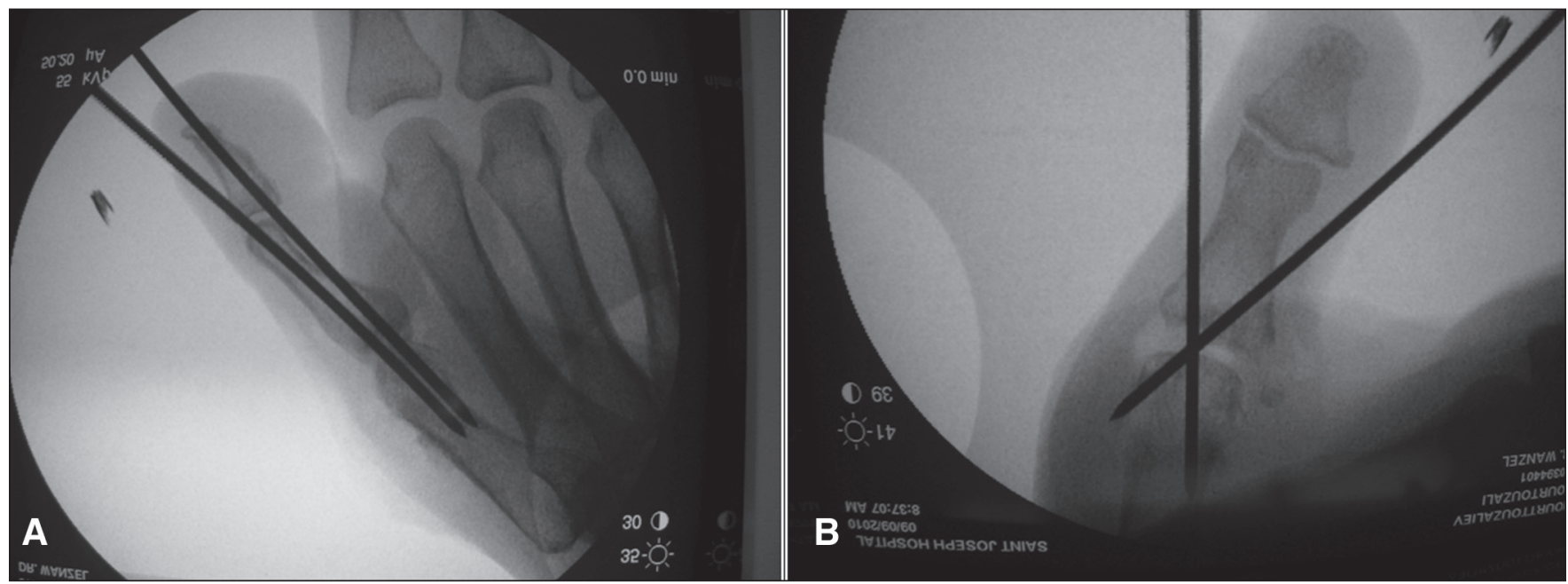

Figure 4) Lateral view (A) and anteroposterior view (B) of intraoperative fluoroscopy after open reduction of the complete complex right thumb metacarpophalangeal joint dislocation showing congruous anatomical reduction of the joint

sesamoid bones, collateral ligaments, bony fragments and the flexor pollicis longus tendon.

Of particular clinical importance in the emergency room or outpatient clinic is the fact that a simple complete MP joint dislocation can be transformed into a complex complete irreducible dislocation if not reduced properly and in a timely manner (7). Closed reduction is best attempted under expertly applied local anesthetic so that the patient is comfortable, relaxed and pain free. For the thumb, we prefer radial and median nerve blocks performed at the wrist. Distally directed pressure is then applied to the base of the proximal phalanx with the metacarpal in a position of flexion and adduction. If necessary, flexing the interphalangeal joint and the wrist will further relax an entrapped flexor pollicis longus tendon that may be blocking reduction. It is imperative not to pull and distract the proximal phalanx when attempting the reduction. This opens up the MP joint space and affords the opportunity for the volar plate to flip over the metacarpal head and become trapped, converting a simple dislocation into one that is complex.

If closed reduction is attempted as described above and is unsuccessful, an operative reduction is required. There is some controversy in the literature regarding the preferred approach to open reduction. Farabeuf (6) first described the dorsal approach, claiming it offers good visualization to release the entrapped volar plate without any risk of injury to neurovascular structures. Kaplan (5) later described the volar approach, concluding that this approach could better address under more direct visualization the anatomical pathology most commonly involved in these irreducible dislocations, namely the volar plate or flexor pollicis longus tendon.

In the volar approach, a Bruner type incision is made on the volar aspect of the MP joint. Care is taken not to damage the displaced and more superficially located neurovascular bundles $(5,19)$. The A1 pulley is released, the flexor pollicis longus tendon is moved radially or ulnarly, the joint is inspected and the offending anatomical structure(s) removed from the joint space under direct visualization.

In the dorsal approach, the extensor apparatus is split longitudinally and the joint approached from a dorsal direction. A trapped interposed volar plate is usually easily identified, split longitudinally and anatomically reduced. Proponents of the dorsal approach cite several advantages. These include lower risk of injury to the digital neurovascular bundles, full visualization of a dorsally entrapped volar plate and, if present, a better management of associated osteochondral fractures $(6,9,11)$. Unfortunately, the dorsal open reduction is also associated with its own drawbacks. It requires vertical splitting of volar plate to reduce it and the metacarpal head. It has been hypothesized that splitting of the volar plate could reduce long-term stability of the MP joint.
The length of time between injury and reduction is also important. Ideally, any dislocated joint should be reduced within hours. The longer it remains dislocated, the harder it is to obtain a closed reduction. The longer a joint remains unreduced, the more likely the development of degenerative arthritis and the less satisfactory the results of surgery in terms of pain, joint stability and range of motion. After several months, contraction of soft tissues (eg, collateral ligaments) occurs, and more 'surgically inflicted' trauma is usually required to obtain satisfactory reduction, again further limiting postoperative expectation of regain of function.

Postoperatively, there is some debate over the period of immobilization. Some authors recommend an early mobilization protocol $(20,21)$, while others prefer immobilization for three to four weeks postoperatively (20). As a guide, it is important to stress the MP joint postreduction to assess the degree of joint stability. If it feels stable, an earlier mobilization protocol is reasonable. If it is unstable, splinting and/or K-wires are required to stabilize the joint, preferably in approximately 25 degrees of flexion. In this instance, most recommend three to four weeks of immobilization followed by range-of-motion exercises guided by a trained hand therapist and gradual weaning of the splint.

Given the late presentation of our patient, we were quite satisfied with the ease with which we were able to obtain an anatomical reduction without too much untoward dissection and destruction of normal structures. However, given the postreduction instability of his joint, his $\mathrm{K}$-wires were left in for three weeks, after which range-of-motion exercises were initiated in our hand therapy department.

\section{CONCLUSION}

An unusual case of a two month late presentation of a complex complete dorsal dislocation of the thumb MP joint approached from a dorsal incision was presented. Awareness and knowledge regarding timeliness of reduction, proper technique of closed reduction and operative approaches to the dislocated MP joint are imperative in the plastic surgery outpatient clinic.

DISCLOSURES: The authors have no financial disclosures or conflicts of interest to declare.

\section{REFERENCES}

1. Lourie GM, Gaston RG, Freeland AE. Collateral ligament injuries of the metacarpophalangeal joints of the fingers. Hand Clin 2006;22:357-64.

2. Zemel NP. Metacarpophalangeal joint injuries in fingers. Hand Clin 1992;8:745-54.

3. Melone CP Jr, Beldner S, Basuk RS. Thumb collateral ligament injuries. An anatomic basis for treatment. Hand Clin 2000;16:345-57. 
4. Posner MA, Retaillaud JL. Metacarpophalangeal joint injuries of the thumb. Hand Clin 1992;8:713-32.

5. Kaplan EB. Dorsal dislocation of the metacarpophalangeal joint of the index finger. J Bone Joint Surg Am 1957;39:1081-6.

6. Farabeuf LH. De la luxation du ponce en arriere. Bull Acad de Chir 1876;2:21.

7. McLaughlin HL. Complex "locked" dislocation of the metacarpophalangeal joints. J Trauma 1965;5:683-8.

8. Johnson AE, Bagg MR. Ipsilateral complex dorsal dislocations of the index and long finger metacarpophalangeal joint. Am J Orthop (Belle Mead NJ) 2005;34:241-5.

9. Becton JL, Christian JD Jr, Goodwin HN, Jackson JG III. A simplified technique for treating the complex dislocation of the index metacarpophalangeal joint. J Bone Joint Surg Am 1975;57:698-700.

10. Barry K, McGee H, Curtin J. Complex dislocation of the metacarpo-phalangeal joint of the index finger: $\mathrm{A}$ comparison of the surgical approaches. J Hand Surg Br 1988;13:466-8.

11. Bohart PG, Gelberman RH, Vandell RF, Salamon PB. Complex dislocations of the metacarpophalangeal joint. Clin Orthop Relat Res 1982:208-10.

12. Sodha S, Breslow GD, Chang B. Percutaneous technique for reduction of complex metacarpophalangeal dislocations. Ann Plast Surg 2004;52:562-5.

13. Patterson RW, Maschke SD, Evans PJ, Lawton JN. Dorsal approach for open reduction of complex metacarpophalangeal joint dislocations. Orthopedics 2008;31:1099.

14. Adler GA, Light TR. Simultaneous complex dislocation of the metacarpophalangeal joints of the long and index fingers. A case report. J Bone Joint Surg Am 1981;63:1007-9.

15. al-Qattan MM, Murray KA. An isolated complex dorsal dislocation of the MP joint of the ring finger. J Hand Surg Br 1994;19:171-3.

16. Hunt JC, Watts HB, Glasgow JD. Dorsal dislocation of the metacarpophalangeal joint of the index finger with particular reference to open dislocation. J Bone Joint Surg Am 1967;49:1572-8.

17. Andersen JA, Gjerloff CC. Complex dislocation of the metacarpophalangeal joint of the little finger. J Hand Surg Br 1987;12:264-6.

18. Miller PR, Evans BW, Glazer DA. Locked dislocation of the metacarpophalangeal joint of the index finger. JAMA 1968;203:300-1.

19. Chadha M, Dhal A. Vulnerability of the radial digital neurovascular bundle of the index finger while using the Kaplan's volar approach for irreducible dislocation of the second metacarpophalangeal joint. Injury 2005;36:126-5.

20. Green DP, Terry GC. Complex dislocation of the metacarpophalangeal joint. Correlative pathological anatomy. J Bone Joint Surg Am 1973;55:1480-6.

21. Eglseder WA Jr, Gens DR, Burgess AR. Multiple ipsilateral dorsal metacarpophalangeal and proximal interphalangeal joint dislocations: A case report. J Trauma 1995;38:955-7. 